\title{
The value of transbronchial lung biopsy using jumbo forceps via rigid bronchoscope in diffuse lung disease
}

\author{
G.L. Casoni1, C. Gurioli1, P.N. Chhajed2, M. Chilosi3, M. Zompatori4, \\ D. Olivieri5, V. Poletti1,5
}

\begin{abstract}
The value of transbronchial lung biopsy using jumbo forceps via rigid bronchoscope in diffuse lung disease. G.L. Casoni, C. Gurioli, P.N. Chhajed, M. Chilosi, M. Zompatori, D. Olivieri, V. Poletti.

Background. Transbronchial lung biopsy (TBLB) is a valuable procedure used to obtain a parenchymal specimen in the evaluation of diffuse lung infiltrates. Large forceps are expected to result in larger specimens and improve diagnostic yield.

Aim. The objective of this study was to evaluate diagnostic yield of TBLB using large modified flexible gastroenterological forceps ("Jumbo forceps") compared with 'normal' flexible forceps via rigid bronchoscopy in patients with diffuse parenchymal lung disease (DPLD).

Methods. The study was a prospective analysis of 95 patients who underwent fluoroscopy guided TBLB over a two year period. Patients with a lung mass or solitary lung nodule undergoing TBLB were excluded. The larger and small forceps were used in a random sequence to avoid a reduction in diagnostic yield of the second series of biopsies related to possible bleeding by first series of biopsies. To minimize the consequence of haemorrhage, we per-
\end{abstract}

\begin{abstract}
formed every rigid bronchoscopy, placing a non inflated Fogarty balloon and a rigid aspirator (diameter $4 \mathrm{~mm}$ ) in lobar bronchus near the biopsy segment. The Fogarty balloon has been inflated in case of bleeding. After the bleeding was controlled we continued to operate up to the biopsy segment.

Results. Diagnostic yield of TBLB using Jumbo forceps was significantly higher than using normal flexible forceps via rigid bronchoscopy in patients with DPLD ( $p=0.001)$. In 74 out of 95 patients $(78 \%)$ the diagnosis was placed with Jumbo forcep while the smaller forcep was diagnostic in 62 out of 95 patients $(65 \%)$. Large forceps obtained significantly more tissue than the small forceps; the biopsy specimen taken with normal forcep measured in average $1.4 \times 1.0 \mathrm{~mm}$ and the larger biopsy taken with jumbo forcep measured in average $2.5 \times 1.9$ mm $(p<0.005)$.

Conclusion. The use of large biopsy forceps to perform TBLB via rigid bronchoscope can significantly increase diagnostic yield in the pathological diagnosis of diffuse infiltrative lung disease.

Monaldi Arch Chest Dis 2008; 69: 2, 59-64.
\end{abstract}

Keywords: Diffuse infiltrative lung diseases, Transbronchial lung biopsy, Rigid bronchoscope, Flexible bronchoscope, High resolution computed tomography.

${ }^{1}$ Dipartimento Toracico, Ospedale GB Morgagni, Forli, Italy.

2 Pulmonary Medicine, University Hospital Basel, Switzerland.

3 Dipartimento di Anatomia Patologica, Università di Verona, Verona, Italy.

${ }^{4}$ Dipartimento di Scienze Cliniche, Sezione Diagnostica per Immagini, Università degli Studi di Parma, Italy.

5 Dipartimento di Scienze Cliniche, Sezione di Clinica Pneumologica, Università di Parma, Parma, Italy.

Correspondence: Venerino Poletti MD, Dipartimento Toracico, Ospedale GB Morgagni, Via Forlanini 34, 47100 Forlì, Italy; e-mail:vepolet@tin.it; v.poletti@ausl.fo.it

\section{Introduction}

Transbronchial lung biopsy (TBLB), refers to the bronchoscopic technique of obtaining pulmonary parenchymal tissue for histologic analysis. Many terms have been used to encompass the large group of disorders that primarily affect the lung parenchyma in a diffuse manner. Diffuse parenchymal lung disease (DPLD) is increasingly favoured worldwide as a generic term for these disorders, rather than terms such as interstitial lung disease or diffuse lung disease. Histological examination should be performed in the majority of cases of DPLD [1-3]. However the question of which kind of biopsy should be performed to diagnose DPLD has a long and controversial history. TBLB achieves a high diagnostic yield in DPLD with centrilobular accentuation such as granulomatous and neoplastic diseases $[4,5]$ but the role of TBLB in diagnosis of idiopathic interstitial pneumonia (IIP) is contentious. Some studies have demonstrated that the morphologic findings of interstitial inflammation and fibrosis in TBLB appeared to have little relevance to subsequent clinical course. However, it has been shown that large forceps are expected to result in larger specimens and to improve the diagnostic yield $[6,7]$. We hypothesised that the use of large forceps via the rigid bronchoscope to perform TBLB in DPLD might increase the diagnostic yield of the bioptic procedure. Therefore the aim of present study was to evaluate diagnostic yield of TBLB using large modified 
flexible gastroenterological forceps compared with "standard" flexible forceps via rigid bronchoscopy in patients with DPLD.

\section{Methods}

The study was a prospective analysis of patients who underwent TBLB between January 2005 and January 2007 at the G.B. Morgagni Hospital of Forlì, Italy. This study was approved by the Institutional Review Board. We defined TBLB as a biopsy performed through a rigid bronchoscope with the intention of obtaining adequate parenchymal lung tissue for meaningful histologic analysis. TBLB performed for a lung mass or solitary pulmonary nodule were excluded. The term diffuse lung disease was used to describe, multi-lobar parenchymal changes with involvement of at least one lower lobe. Procedures were performed or supervised by a group of experienced and dedicated bronchoscopists. Prior to TBLB, the patients were evaluated in a routine fashion with a clinical history, physical examination, and chest radiograph. Lesions from which biopsy specimens were to be taken were localised and characterised by highresolution computed tomography (HRCT).

Rigid bronchoscopy was performed under general anesthesia in the operating room. Patients were oxygenated by mask and pharyngeal secretions were aspirated before intubation. Dentures were removed, and the teeth and gums were carefully inspected. Folded gauze, foam rubber, or plastic mouth guards were used to protect these structures from damage during rigid bronchoscopy. Topical anesthesia was administered with lidocaine $2 \%$ before intubation. General anesthesia was induced using propofol (1-2 mg/kg). Assisted spontaneous ventilation was applied. After anesthesia induction, the patients were intubated with a rigid bronchoscope or tracheoscope (Storz; Tutlingen, Germany). Anesthesia was maintained with intravenous propofol (4 to $6 \mathrm{mg} / \mathrm{kg} / \mathrm{h}$ ) according to the hemodynamic parameters of the patients. The fraction of inspired oxygen administered ranged between 0.35 and 1.0, and was adjusted based on transcutaneous oxygen saturation values.

All transbronchial biopsies were performed in lower lobes using fluoroscopic guidance. This study compared the diagnostic yield of transbronchial biopsies using large and small forceps: a Jumbo forcep with cup size 9 × 3 × $3 \mathrm{~mm}$ (Model K 011340, Diflex, Germany) and a 'normal' flexible forceps (Model K 022V-120, Diflex, Germany) with cup sizes, $3 \times 2 \times 0.9 \mathrm{~mm}$ (fig. 1). Each patient had a biopsy using both forceps in the same lobe. For each patient the number of fragments obtained were 10 (5 obtained fragments with jumbo forceps and 5 with normal flexibile forceps). The larger and small forceps were used in a random sequence to avoid a reduction in diagnostic yield of the second series of biopsies related to possible bleeding by first series of biopsies.

To minimize the consequence of hemorrhage, we performed every rigid bronchoscopy, placing a non inflated Fogarty balloon and a rigid aspirator (diameter $4 \mathrm{~mm}$ ) in lobar bronchus near the biopsy segment. The Fogarty balloon was inflated in case of bleeding. After the bleeding was controlled we continued to perform up to ten biopsies. Biopsy specimens were placed in a container with formaldehyde solution. Specimens were then embedded in paraffin, sectioned, placed on a separate slide marked with its identifying label, and stained with hematoxylin-eosin. Special stains were performed as necessary for each case. Diagnostic yield was compared by a pathologist, blinded to the size of forceps used on the basis of the relative amount of tissue obtained and ability to ascertain a histopathologic diagnosis.

\section{Statistical Analysis}

Group data was expressed as mean (SE) values. Statistically significant differences between patients with and without diagnosis and differences between groups were evaluated by either McNemar's or Student's $t$ test. A $p$ value of $<0.05$ was considered statistically significant.

\section{Results}

A total of 95 patients underwent TBLB via rigid bronchoscope for evaluation of DPLD during the time period of this study. The baseline characteristics and lung function parameters of patients are shown in table 1. Diagnostic yield of TBLB using Jumbo forceps was significantly higher than using normal flexible forceps via rigid bronchoscopy in patients with diffuse parenchymal lung disease (DPLD) $(p=0.001)$. In 74 out of 95 patients $(78 \%)$ the diagnosis was placed with Jumbo forcep while the smaller forcep was diagnostic in 62 out of 95 patients $(65 \%)$. Large forceps obtained significantly more tissue than did small forceps; the biopsy specimen taken with normal forcep measured in average 1.4 x $1.0 \mathrm{~mm}$ and the larger biopsy taken with jumbo forcep measured in average $2.5 \times 1.9$ $\mathrm{mm}(p<0.005$,$) . TBLB had a high diagnostic yield$ in disorders that significantly involve terminal and

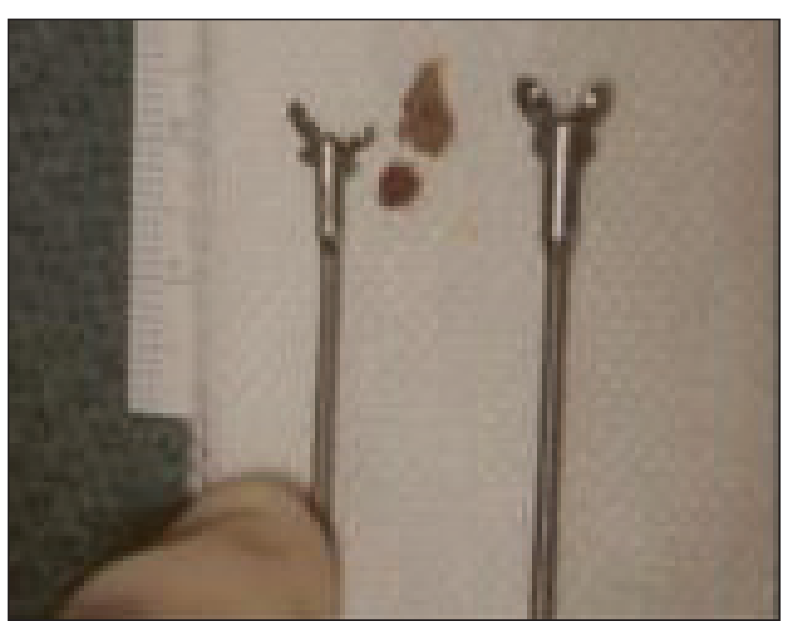

Fig. 1. - Difference between big bioptic forceps on the right (Jumbo forceps) used via rigid bronchoscope with a lung sample that is poured out of the tip of the forceps and standard flexible bioptic forceps for fiber-bronchoscope. 
Table 1. - Baseline and clinic-functional characteristics of DPLD patients

\begin{tabular}{lc}
\hline Age $(\mathrm{yr}) \pm \mathrm{DS}$ & $52 \pm 1.2$ \\
$\mathrm{Sex}(\mathrm{M} / \mathrm{F})$ & $63 / 32$ \\
Smoking history & \\
(current, former, never smokers) & $11 / 58 / 26$ \\
$\mathrm{FEV}_{1}(\mathrm{~L}) \pm \mathrm{DS}$ & $2.7 \pm 0.05$ \\
$\mathrm{FVC}(\mathrm{L}) \pm \mathrm{DS}$ & $3.44 \pm 1.6$ \\
$\mathrm{DLCO}(\%$ pred $) \pm \mathrm{DS}$ & $62.8 \pm 4.2$ \\
$\mathrm{PaO}_{2}(\mathrm{mmHg}) \pm \mathrm{DS}$ & $69.2 \pm 1.2$ \\
$\mathrm{PaCO}_{2}(\mathrm{mmHg}) \pm \mathrm{DS}$ & $38.3 \pm 0.5$ \\
$\mathrm{pH}$ & $7.41 \pm 0.03$ \\
$* \mathrm{P}<0.05$ & \\
\end{tabular}

respiratory bronchioles (12 cases of organising pneumonia and 6 cases of hypersensitivity pneumonitis) or distributed along the lymphatic routes, ( 8 cases of sarcoidosis) but Jumbo TBLB was useful also in 8 cases of cellular non specific interstitial pneumonia (NSIP) pattern. A confirmatory surgical biopsy was done only in the two cases with no identifiable cause (idiopathic NSIP) and in the remaining 6 patients a specific clinical setting (Dermatomyositis/Polymiositis in 2 cases; Sjogren Syndrome 2 cases; mixed collagen vascular disease 2 patients) was present and hence no further biopsy investigations were deemed necessary. In 21 of the 95 patients $(22 \%)$, biopsies performed resulted in non-specific diagnoses, including fibrosis or chronic inflammation. Video assisted thoracoscopic biopsy (VATS) was performed in 17 out of these 21 patients. The main category of pathologic diagnoses obtained with VATS was IIP, in particular usual interstitial pneumonia (UIP) (7 cases) and fibrosing variant of NSIP (5 cases). Four patients in whom TBLB was not diagnostic refused further invasive diagnostic procedures. The categories of pathologic diagnoses using large forceps, smaller forcep and thoracoscopic biopsy are shown in tables 2, 3 and 4. No difference was observed in the amount of postbiopsy bleeding with either forceps [minor bleeding $<200 \mathrm{ml}$ in 7 out of 95 patients (in 3 pts with small forcep and in $4 \mathrm{pt}$ with Jumbo forceps)]. The main procedure related complication was pneumothorax observed in four patients (4\%) (but only a 1 patient was treated with drainage tube).

\section{Discussion}

This study prospectively compared the diagnostic yield of transbronchial biopsies using large and small forceps in diagnosis of DPLP. Our findings suggest that the use of large biopsy forceps to perform TBLB via rigid bronchoscope can significantly increase diagnostic yield in the pathological diagnosis of DPLP.

Table 2. - Etiology of diagnostic TBLB with Jumbo forceps

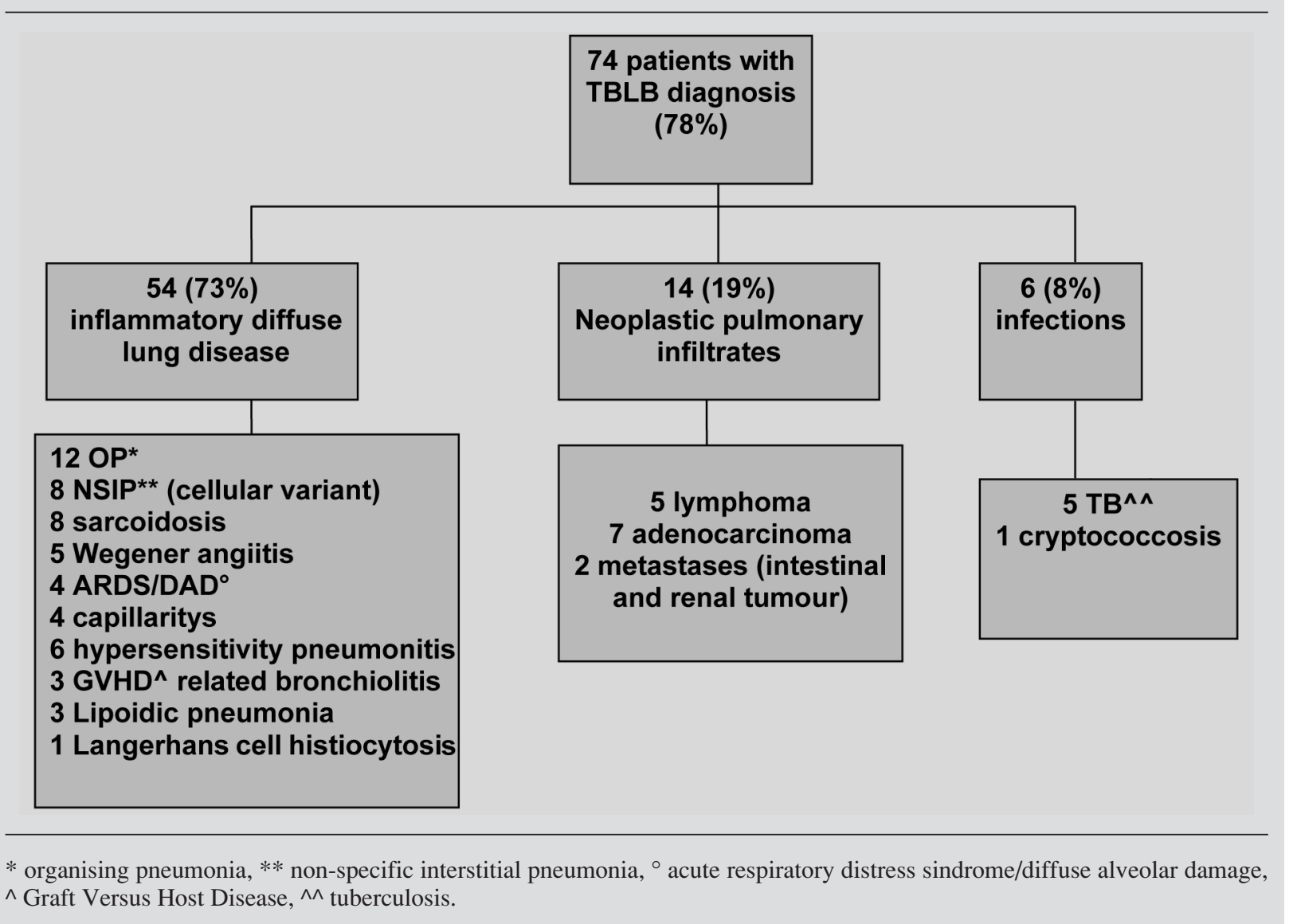


Table 3. - Etiology of diagnostic TBLB with small forceps

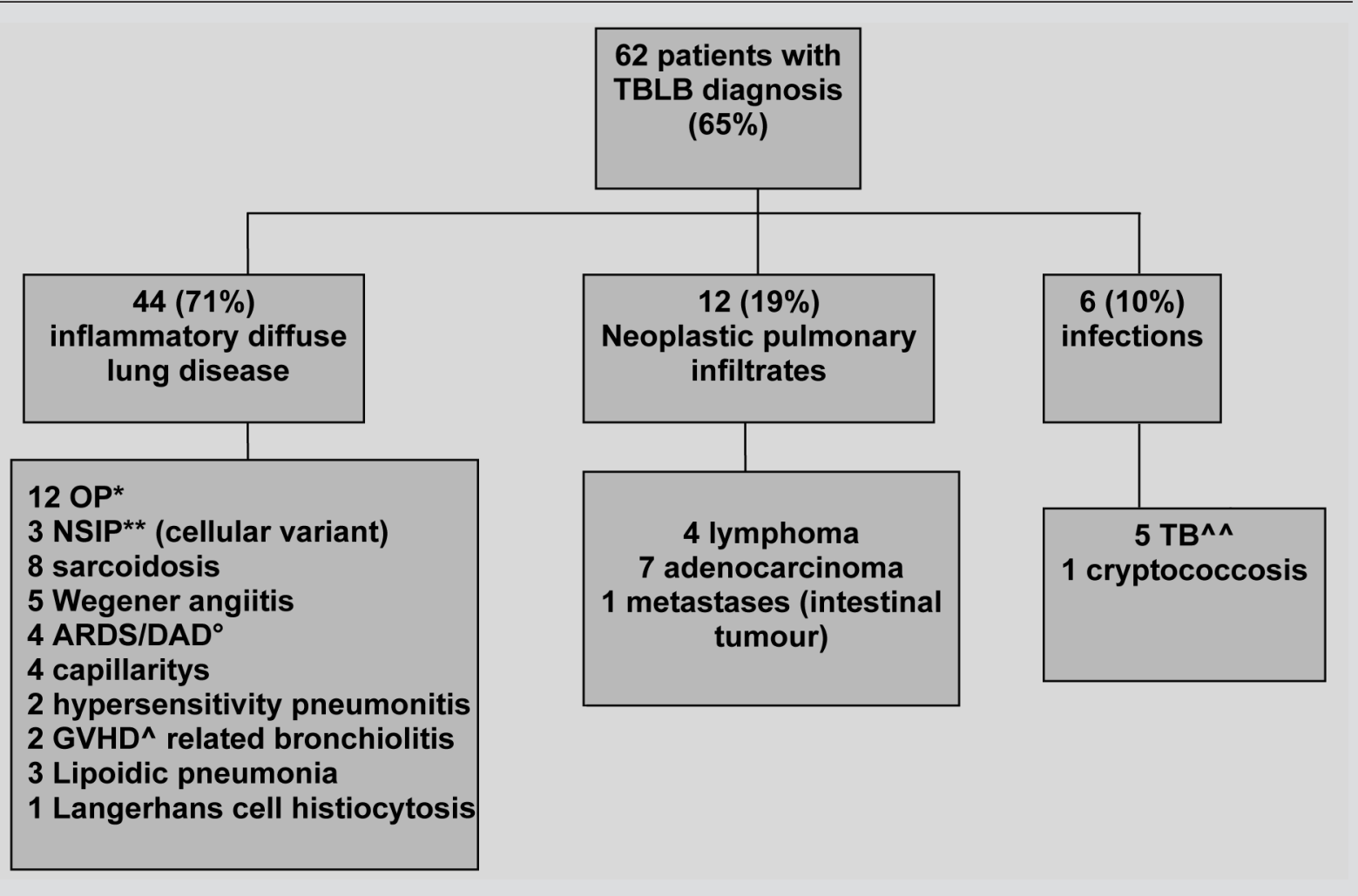

$*$ organizing pneumonia, $* *$ non-specific interstitial pneumonia, ${ }^{\circ}$ acute respiratory distress sindrome/diffuse alveolar damage, $\wedge$ Graft Versus Host Disease, $\wedge \wedge$ tuberculosis.

Table 4. - Final etiology (VATS lung biopsy was performed) of patients with no diagnostic TBLB

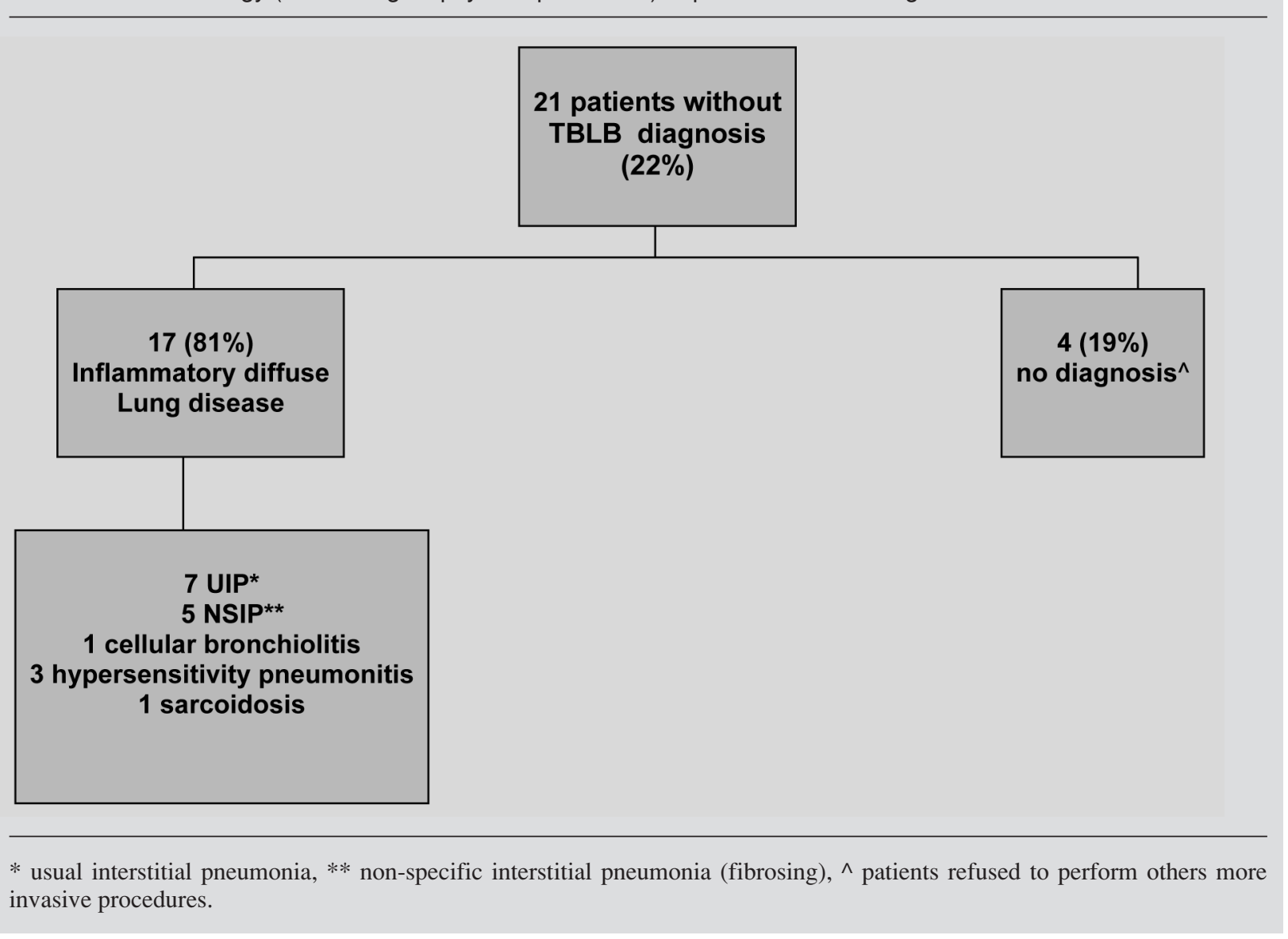


The disorders that are centred around terminal and respiratory bronchioles (respiratory bronchiolitis, tuberculosis, lobular infectious pneumonia, cellular bronchiolitis) or significantly involve these structures (organizing pneumonia) or are distributed along the lymphatic routes (sarcoidosis, carcinomatous lymphangitis) may be easily sampled by the forceps $[5,8]$. However, it has become widely accepted that TBLB is not useful in diagnosing IIP, despite a lack of convincing evidence and recent studies suggesting that UIP pattern may also be identified in small specimens $[9,10]$.

Large forceps obtained significantly more tissue than did small forceps, however the Jumbo forceps are too big to be introduced through a flexible bronchoscope; therefore it is necessary to use the rigid bronchoscope. As published by Berbesku E. et al. [9] a common problem encountered in interpreting TBLB specimens of interstitial lung disease is the frequent presence of artifactual atelectasis that may both obscure diagnostic features and also be interpreted incorrectly as interstitial fibrosis. The biopsy forceps in the technique described in this manuscript are not passed through the operating channel of the flexible bronchoscope and therefore the samples, frequently larger than the tip of the forceps, are not squeezed and hence crush artefacts may be avoided.

The significant higher diagnostic yield using Jumbo forceps via rigid bronchoscope may be explained by the fact that large forceps are expected to result in larger specimens. A study [6] reported that larger transbronchial lung biopsies were more likely to contain diagnostic tissue while Loube et al. [7] prospectively compared the diagnostic yield of TBLB using large and small forceps (cup sizes, $3 \times 2 \times 0.9$ vs. 2 × $1.5 \times 0.6 \mathrm{~mm}$ respectively) proving that large forceps obtained significantly more tissue than did small forceps. This is an important issue because the possibility to study pathology of pulmonary disease in large detail can increase greatly the diagnostic yield of procedure. However, the knowledge of the clinical context, radiologic distribution of abnormalities, and histopathologic patterns is essential for diagnosis of DLPD. Further, a very nice study [11] focused on how TBLB results were clinically useful in the management of patients with DLPD and it indicated that TBLB was a clinically useful test in about $75 \%$ of procedures. Therefore this bioptic procedure should be proposed in diagnostic "algorithm" of DPLD in particular to avoid, if possible, the VATS. Indeed, one criticism of this procedure is that the specimens can be performed almost exclusively in the lower lobes but in the "methods" of this study the term diffuse lung disease was used to describe, multi-lobar parenchymal changes with involvement of at least one lower lobe. In patients where the TBLB using large forceps was not diagnostic, VATS was performed. The main category of pathologic diagnoses obtained with VATS was IIP, in particular UIP and fibrosing variant of NSIP. Although this data suggests that TBLB using larger flexible forceps have a limited role in the pathological diagnosis of UIP and fibrosing variant of
NSIP [12] (using larger flexible forceps too), however we found that generous TBLB may provide good specimens for a morphological diagnosis of cellular variant NSIP. This pathological diagnosis is acceptable in specific clinical settings (polymyositis-dermatomyositis-related interstitial lung disease, drug toxicity) but it might also be of clinical value in cases of unknown etiology [13]. In eight patients in which a NSIP cellular pattern was documented on TBLB specimens, a confirmatory surgical biopsy was deemed necessary only in the two patients in whom no identifiable cause were found (idiopathic NSIP). In the remaining six patients with cellular variant of NSIP, the need for surgical confirmation was precluded due to the presence of a specific clinical setting (Dermatomyositis/Polymiositis in 2 cases; Sjogren Syndrome 2 cases; mixed collagen vascular disease 2 patients) $[11,13]$. Finally, no difference was observed in the amount of post-biopsy bleeding with either forceps [minor bleeding $<200 \mathrm{ml}$ in 7 out of 95 patients (in 3 pts with small forceps and in $4 \mathrm{pt}$ with Jumbo forceps)]. The main procedure related complication was pneumothorax observed in four patients (4\%) (but only a 1 patient was treated with drainage tube). Assessing the low percentages of procedure related complications, we believe that TBLB using Jumbo forceps may also be performed safely in patients with clinical and radiographic features compatible with DPLD.

In conclusion, in our study we have shown that characteristic histologic features of DPLD can be identified on TBLB specimens more often than previously appreciated using large modified flexible forceps via rigid bronchoscopy [5] probably because large forceps obtained significantly more tissue than small forceps increasing diagnostic yield of procedures. On one hand our data confirms that TBLB have a high diagnostic yield in disorders that significantly involve terminal and respiratory bronchioles or distributed along the lymphatic routes confirming, while on other hand it demonstrates that generous TBLB using larger flexible forceps (Jumbo forceps) may also be useful to obtain specimens of bigger dimensions to place a morphologic diagnosis in cellular NSIP pattern. The knowledge of the clinical context, radiological distribution of abnormalities, and histopathologic patterns is essential for diagnosis of DLPD. Only with this information, can the pathologist substantially influence the diagnostic workup and help guide the clinician to an accurate clinical/radiologic/pathologic diagnosis.

It is important that larger and more detailed studies are conducted to confirm these results and to establish the method of taking biopsy specimens with the best yield for studying the pathologic condition of DPLD and to minimize possibly the need to refer patients to diagnostic VATS.

Conflict of Interest Statement: None of the authors have a conflict of interest to declare in relation to this work under this sub-heading or any financial and personal relationships with other people or organisations that could inappropriately influence (bias) their work. 


\section{References}

1. Crystal RG, Fulmer JD, Roberts WC, et al. Idiopathic pulmonary fibrosis: clinical, histological, radiographic, physiologic, scintigraphic, cytologic and biochemical aspects. Ann Intern Med 1976; 85: 769-788.

2. Turner-Warwick M. Infiltrative and interstitial lung disease. In: Brewis RAL, Gibson GJ, Geddes DM, eds. Respiratory medicine. London: Bailliere Tindall, 1990.

3. British Thoracic Society, Standards of Care Committee. The Diagnosis, Assessment and Treatment of Diffuse Parenchymal Lung Disease in Adults. Thorax 1999; 54 (Suppl 1): S1-S28.

4. Becker HD, Shirakawa T, Tanaka F, Muller KM, Herth F. Transbronchial (transbronchoscopic) lung biopsy in immunocompromised patient; in Strausz J (ed): Pulmonary Endoscopy and Biopsy Techniques. Eur Respir Monogr 1998, vol 3, pp 193-208.

5. Poletti V, Patelli M, Ferracini R, Simonetti M, Spiga L. Transbronchial lung biopsy in infiltrative lung disease. The importance of the pathologic approach. Sarcoidosis 1988; 5: 43-50.

6. Curley FJ, Johal JS, Burke ME, Fraire AE. Transbronchial lung biopsy: Can specimen quality be predicted at the time of biopsy? Chest 1998; 113: 1037-1041.
7. Loube DI, Johnson JE, Wiener D, Anders GT, Blanton HM, Hayes JA. The effect of forceps size on the adequacy of specimens obtained by transbronchial biopsy. Am Rev Respir Dis 1993; 148: 1411-1413.

8. Ryu JH, Olson EJ, Midthun DE, Swensen SJ. Diagnostic approach to the patient with diffuse lung disease. Mayo Clin Proc 2002; 77: 1221-1227.

9. Berbesku EA, Katzenstein AL, Snow JL, et al. Transbronchial biopsy in usual interstitial pneumonia.Chest 2006; 129: 1126-1131.

10. Wall CP, Gaensler EA, Carrington CB, et al. Comparison of transbronchial lung biopsy and open biopsies in chronic infiltrative lung diseases. Am Rev Respir Dis 1981, 123: 280-285.

11. Ensminger SA, Prakash UB. Is bronchoscopic lung biopsy helpful in the management of patients with diffuse lung disease? Eur Respir J 2006; 28: 1081-4.

12. Andersen HA, Fontana RS, Harrison EG, Jr. Transbronchoscopic Lung Biopsy in Diffuse Pulmonary Disease. Dis Chest 1965; 48: 187-192.

13. Watanabe K, Higuchi K, Ninomiya K, Ohshima T, Aritomi T, Kawabata Y, Yoshida M. Steroid treatment based on the findings of transbronchial biopsy in idiopathic interstitial pneumonia. Eur Respir $J$ 2002; 20: 1213-9.

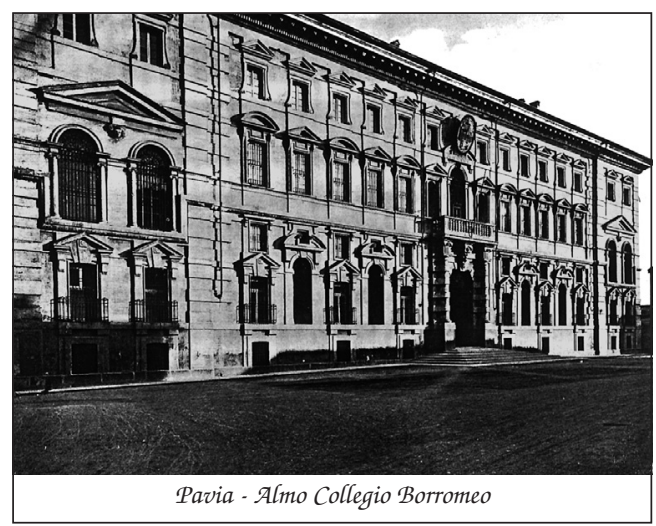

